\title{
Extended versus point light source: where does the difference in the illuminance exist?
}

\author{
V. Ivchenko \\ Department of Natural Sciences Training, \\ Kherson State Maritime Academy, Kherson 73000, Ukraine \\ e-mail: reterty@gmail.com
}

Received 23 June 2020; accepted 30 September 2020

\begin{abstract}
In this paper we derive and analyse the expressions to find the illuminance from luminous ball, disc and line in the case of general position of the light receiver. We show that one can always replace a luminous ball with a point light source located at its center and having the appropriate luminous intensity. Any luminous disc or line can be considered, with reasonable accuracy (the relative error in the determination of the illuminance is less than $5 \%$ ), as the point light source with anisotropic (cosine) luminous intensity and placed at their center, if the distance to the observation point is approximately four times larger than their characteristic sizes. The issues outlined in this article will be useful for undergraduate students, who study the basics of photometry.
\end{abstract}

Keywords: Illuminance; luminous ball; disc and line .point light source; inverse square law.

DOI: https://doi.org/10.31349/RevMexFisE.18.127

\section{Introduction}

Light sources play an important role in our everyday life. A light source means any object that emits electromagnetic energy in the visible spectrum. Depending on their nature, they are divided into artificial and natural light sources. Depending on the relationship between their characteristic dimensions and the distance to the radiation receiver, all light sources are divided into extended (continuous) and point sources. Most volumetric light sources (spherical incandescent light bulb, the milk glass orb lamps, etc.) can be modelled with a luminous ball. Many flat lamps are well described by the luminous disk model. Finally, fluorescent tubes can be successfully approximated by a luminous line.

An important characteristic of the illuminated surface is the illuminance [1]. There are certain standards for the nominal illuminance of workplaces and premises [2]. That is why it is important to be able to calculate this value from various light sources. In this paper we derive and analyse the expressions to find the illuminance from luminous ball, disc and line in the case of general position of the light receiver. We compare these results with those obtained on the basis of the model of the point light source. This helps the readers to probe the limits of applicability of the point light source model (that is, the limits of applicability of the inverse square law approximation [3]). The issues outlined in this article will be useful for undergraduate students, who study the basics of photometry.

\section{The luminous ball}

Let us consider the luminous ball of radius $R$, which has a uniform luminance $L$, that is, it obeys the Lambert's cosine law [4]. We place infinitesimal illuminated area $\mathrm{d} A^{\prime}$ on one of the symmetry axis of the ball perpendicular to it. According to the definition [1,5], illuminance $E$ is given by relation

$$
E=\frac{\mathrm{d} \Phi}{\mathrm{d} A^{\prime}},
$$

where $\mathrm{d} \Phi$ is the total luminous flux incident on this area. On the other hand [2]

$$
\mathrm{d} \Phi=L \int_{A} \cos \theta \mathrm{d} \Omega^{\prime} \mathrm{d} A,
$$

where $\mathrm{d} A$ is the infinitesimal area of the light source; $\theta$ is the angle between the normal to surface $\mathrm{d} A$ and the direction to $\mathrm{d} A^{\prime} ; \mathrm{d} \Omega^{\prime}$ is the infinitesimal solid angle containing this direction. But

$$
\begin{aligned}
\mathrm{d} \Omega^{\prime} & =\frac{\mathrm{d} A^{\prime}}{l^{2}} \cos \theta^{\prime}, \\
\mathrm{d} \Omega & =\frac{\mathrm{d} A}{l^{2}} \cos \theta,
\end{aligned}
$$

where $l$ is the distance between $\mathrm{d} A$ and $\mathrm{d} A^{\prime} ; \theta^{\prime}$ is the angle between the normal to $\mathrm{d} A^{\prime}$ and the direction of the light; $\mathrm{d} \Omega$ is the solid angle, at which radiant area $\mathrm{d} A$ is visible from the illuminated area $\mathrm{d} A^{\prime}$.

Using Eqs. (1)-(4) we get

$$
E=L \int \cos \theta^{\prime} \mathrm{d} \Omega .
$$

Since $\Omega=2 \pi\left(1-\cos \theta^{\prime}\right)$ [6], we have $\mathrm{d} \Omega=2 \pi \sin \theta^{\prime} \mathrm{d} \theta^{\prime}$. Then, using Eq. (5) we derive:

$$
E=\pi L \int_{0}^{\Theta} \sin 2 \theta^{\prime} \mathrm{d} \theta^{\prime}=\pi L \sin ^{2} \Theta .
$$




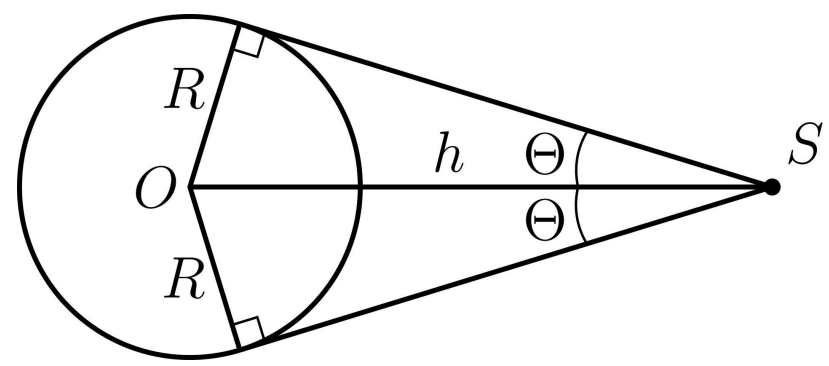

FIGURE 1. Geometry used to derive Eq. (7).

Here $\Theta$ is the angle, at which the edge of the ball is visible from $\mathrm{d} A^{\prime}$. We note that Eq. (6) is applicable not only to a ball, but also to an arbitrary shape light source with a visible boundary in the form of a circle, if the radiation receiver lies on its symmetry axis. For example, it is valid for points lying on the symmetry axis of the luminous disk [7]. It can be also applied for an infinite radiant plane. In this case $\Theta=\pi / 2$ and $E=\pi L=$ const.

Using Eq. (6) and Fig. 1 we immediately obtain for the ball:

$$
E=\frac{\pi L R^{2}}{h^{2}}
$$

where $h$ is the distance between the center of the ball and $\mathrm{d} A^{\prime}$.

Therefore, for any arbitrary distance $h$ the inverse square law takes place. It means that we can always replace a luminous ball with a point light source located at its center and having luminous intensity $I=\pi L R^{2}$.

\section{The luminous disc}

Let us consider the luminous disc of radius $R$, which has a uniform luminance $L$. We investigate the important case, when area $\mathrm{d} A^{\prime}$ is parallel to the disc plane. Then $\theta=\theta^{\prime}$ and using Eqs. (1)-(3) we get

$$
E=L \int_{A} \frac{\cos ^{2} \theta}{l^{2}} \mathrm{~d} A .
$$

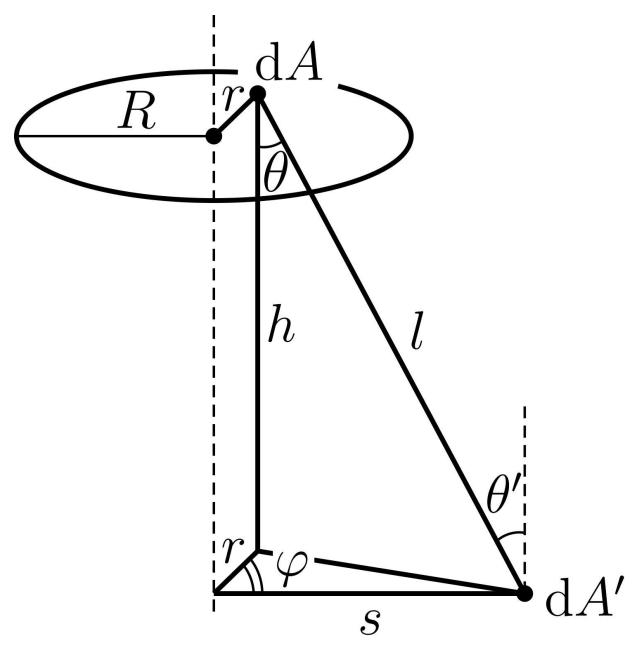

FIGURE 2. Geometry used to derive Eq. (9).

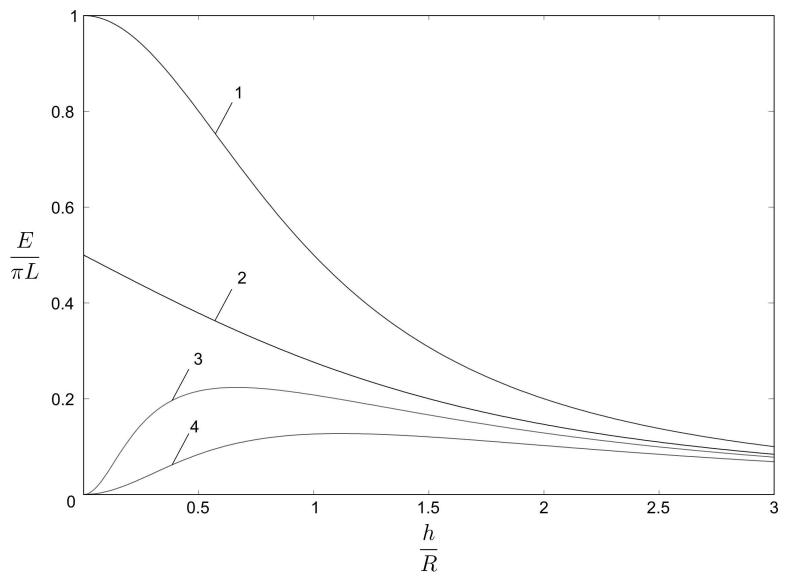

FIGURE 3. Illuminance $E$ from disc as a function of $h$ for different values of $s$. 1) $s=0$;2) $s=R$; 3) $s=1.2 R$;4) $s=1.5 R$.

We set the position of element $\mathrm{d} A$ in the disk plane by $r$ (the current radius) and $\varphi$ (the polar angle). In such a case $\mathrm{d} A=r \mathrm{~d} r \mathrm{~d} \varphi$. Due to the symmetry of the problem, the general position of element $\mathrm{d} A^{\prime}$ is defined by $h \geq 0$ (the shortest distance from $\mathrm{d} A^{\prime}$ to the disc plane) and $s$ (the shortest distance from $\mathrm{d} A^{\prime}$ to the perpendicular symmetry axis of the disc; we allow $s$ to change from $-\infty$ to $\infty$ ). Considering Fig. 2 and applying the law of cosines we write $\cos \theta=h / l$, $l^{2}=h^{2}+r^{2}+s^{2}-2 s r \cos \varphi$.

Using these relations, Eq. (8) and the symmetry of the problem we have

$$
E=2 L h^{2} \int_{0}^{R} r \mathrm{~d} r \int_{0}^{\pi} \frac{\mathrm{d} \varphi}{\left(h^{2}+r^{2}+s^{2}-2 s r \cos \varphi\right)^{2}}
$$

Performing integration in Eq. (9) we finally get

$$
E=\frac{\pi L}{2}\left[1+\frac{R^{2}-h^{2}-s^{2}}{\sqrt{\left(h^{2}+(R-s)^{2}\right)\left(h^{2}+(R+s)^{2}\right)}}\right] .
$$

As expected, along the disk plane $(h=0) E=\pi L$ for $|s|<R$ and $E=0$ for $|s|>R$. Moreover, function $E(h, s)$ is invariant under transformation $s \rightarrow-s$, which is consistent with the symmetry of the problem. At fixed value of $s$ and $|s|<R, E$ is a monotonically decreasing function of $h$, whereas for $|s|>R$ function $E(h)$ has a maximum. As $|s|$ increases, this maximum shifts to the region of large values of $h$; wherein the maximum value of $E$ becomes smaller (Fig. 3).

At fixed value of $h, E$ is a monotonically decreasing function of $|s|$. Using the Taylor series of function $E(R)$ (see Eq. (10)) containing only the first nonzero term, we find the illuminance of point light source corresponding to this extended light source and placed at its center,

$$
E_{\text {point }}=\frac{\pi L R^{2} h^{2}}{\left(s^{2}+h^{2}\right)^{2}} \text {. }
$$




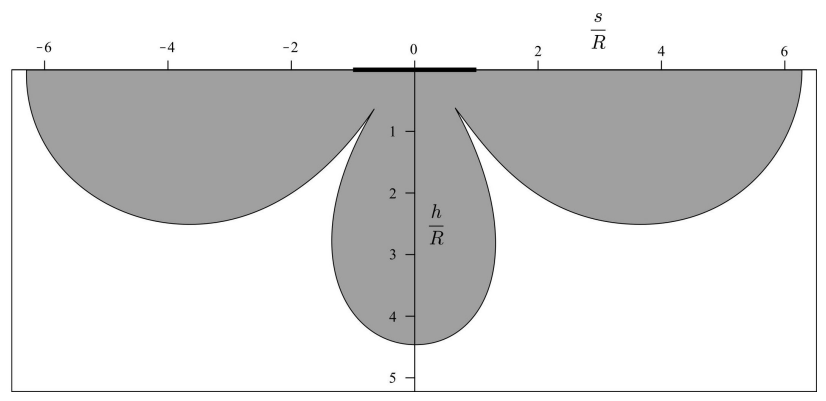

FIGURE 4. Domains of credibility (white) and incredibility (gray) of the point source approximation for the luminous disc at $\delta_{E}=$ $5 \%$.

The illuminance from such a source obeys the inverse square law, but with anisotropic (cosine) luminous intensity: $E=$ $I \cos \alpha /\left(h^{2}+s^{2}\right)$, where $I=I_{0} \cos \alpha, I_{0}=\pi L R^{2}, \alpha=$ $h / \sqrt{h^{2}+s^{2}}$.

Equations (10) and (11) allow one to highlight the domain on plane $(h, s)$, for which Eq. (11) gives approximately correct value for the illuminance from disc. In Fig. 4 we present the results of numerical calculation of contour line, along which the relative error $\delta_{E}=\left|E-E_{\text {point }}\right| / E$ in the determination of image distance is equal to $5 \%$.

The domain corresponding to a larger value of this quantity is shaded in gray.

It is seen that the spatial distribution of the relative error has an anisotropic character. The greatest deviations from the point light source model take place along the disc plane and its perpendicular symmetry axis. We conclude that any luminous disc can be considered, with reasonable accuracy (the relative error in the determination of the illuminance is less than 5\%), as point light source, if the distance to the observation point is approximately three times larger than the disc diameter.

\section{The luminous line}

Let us consider the luminous line of length $2 a$. We introduce constant specific luminous intensity $K$ equal to luminous intensity per unit length of the luminous line and directed along the normal to it. Then, the luminous intensity of infinitesimal line segment $\mathrm{d} x$ is as follows:

$$
\mathrm{d} I=K \mathrm{~d} x \cos \theta,
$$

where $\theta$ is the angle between the normal to $\mathrm{d} x$ and the direction to $\mathrm{d} A^{\prime}$. The illuminance from this segment is given by relation

$$
\mathrm{d} E=\frac{\mathrm{d} I}{l^{2}} \cos \theta^{\prime} .
$$

As in the previous section, we assume that area $\mathrm{d} A^{\prime}$ is parallel to the line, that is, $\theta=\theta^{\prime}$. We set the position of element $\mathrm{d} x$ along the line by coordinate $x$ (the distance from $\mathrm{d} x$ to the line center). The general position of element $\mathrm{d} A^{\prime}$ is defined by $h \geq 0$ (the shortest distance from $\mathrm{d} A^{\prime}$ to the line direction) and $s$ (the shortest distance from $\mathrm{d} A^{\prime}$ to the perpendicular symmetry axis of the line; we allow $s$ to change

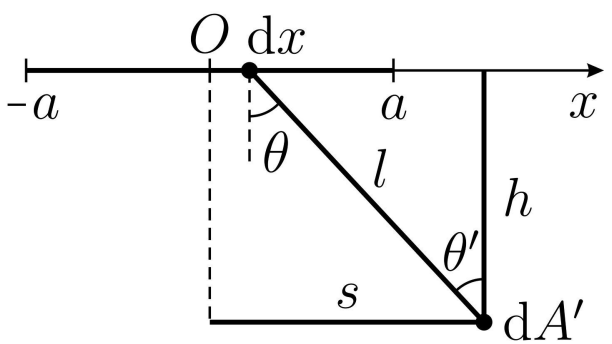

FIGURE 5. Geometry used to derive Eq. (14).

from $-\infty$ to $\infty)$. Considering Fig. 5 we write: $\cos \theta=h / l$, $l^{2}=h^{2}+(s-x)^{2}$.

Using these relations and Eq. (8) we have:

$$
E=K h^{2} \int_{-a}^{a} \frac{\mathrm{d} x}{\left(h^{2}+(s-x)^{2}\right)^{2}} .
$$

Performing integration in Eq. (12) we finally get

$$
\begin{aligned}
E & =K\left[\frac{\arctan \left(\frac{a-s}{h}\right)+\arctan \left(\frac{a+s}{h}\right)}{2 h}\right. \\
& \left.+\frac{a\left(a^{2}+h^{2}-s^{2}\right)}{\left(a^{2}+h^{2}+s^{2}\right)^{2}-4 a^{2} s^{2}}\right] .
\end{aligned}
$$

It follows from Eq. (15) that for $h \rightarrow 0$ and $|s|<R$ $E \rightarrow \infty$. This non-physical result is explained by neglecting the thickness of a given light source. Therefore, Eq. (15) is not applicable at distances from the receiver to the light source of the order of its thickness. For $h=0$ and $|s|>a$ $E=0$. Function $E(h, s)$ is invariant under transformation $s \rightarrow-s$, which is consistent with the symmetry of the problem. At fixed value of $s$ and $|s|<a, E$ is a monotonically decreasing function of $h$ (Fig. 6).

For $|s|>a$ function $E(h)$ has a maximum. As $|s|$ increases this maximum shifts to the region of large values of $h$; wherein the maximum value of $E$ becomes smaller (Fig. 7).

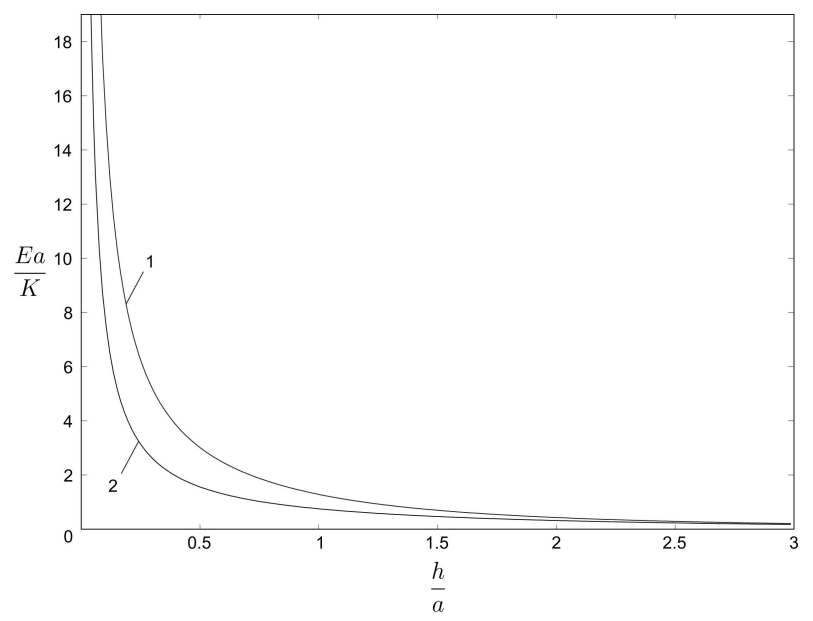

FIGURE 6. Illuminance $E$ from line as a function of $h$ for different values of $s<a$. 1) $s=0$;2) $s=a$. 


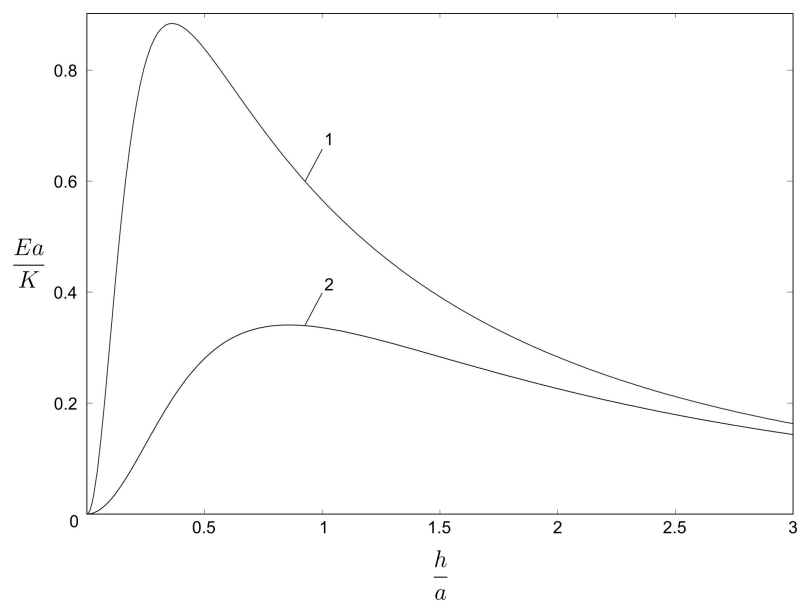

FIGURE 7. Illuminance $E$ from line as a function of $h$ for different values of $s>a$. 1) $s=1.2 a$;2) $s=1.5 a$.

At fixed value of $h, E$ is a monotonically decreasing function of $|s|$.

Using the Taylor series of function $E(a)$ (see Eq. (15)) containing only the first nonzero term, we find the illuminance of point light source corresponding to this extended light source and placed at its center:

$$
E_{\text {point }}=\frac{2 K a h^{2}}{\left(s^{2}+h^{2}\right)^{2}}
$$

The illuminance from such a source obeys the inverse square law, but with anisotropic (cosine) luminous intensity: $E=$ $I \cos \alpha /\left(h^{2}+s^{2}\right)$, where $I=I_{0} \cos \alpha, I_{0}=2 K a, \alpha=$ $h / \sqrt{h^{2}+s^{2}}$.

Equations (15) and (16) allow one to highlight the domain on plane $(h, s)$, for which Eq. (11) gives approximately correct value for the illuminance from the disc. In Fig. 8 we present the results of numerical calculation of contour line, along which the relative error $\delta_{E}=\left|E-E_{\text {point }}\right| / E$ in the determination of image distance is equal to $5 \%$.

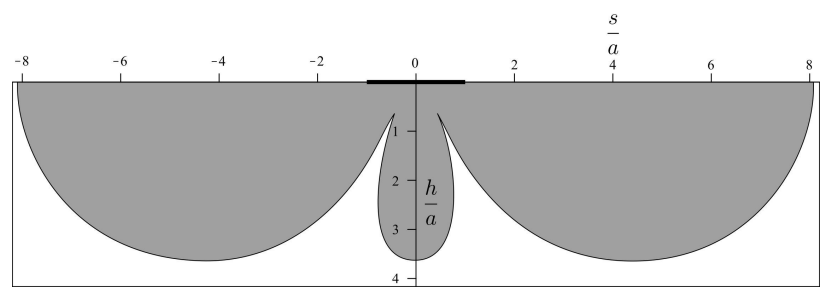

FIGURE 8. Domains of credibility (white) and incredibility (gray) of the point source approximation for the luminous line at $\delta_{E}=$ $5 \%$.

The domain corresponding to a larger value of this quantity is shaded in gray.

It is seen that the spatial distribution of the relative error has in anisotropic character. The greatest deviations from the point light source model take place along the disc plane and its perpendicular symmetry axis. We conclude that any luminous line can be considered, with reasonable accuracy (the relative error in the determination of the illuminance is less than 5\%), as point light source, if the distance to the observation point is approximately four times larger than the line length.

\section{Conclusions}

In this paper we derive and analyse the expressions to find the illuminance from luminous ball, disc and line in the case of general position of the light receiver. We show that one can always replace a luminous ball with a point light source located at its center and having the appropriate luminous intensity. Any luminous disc or line can be considered, with reasonable accuracy (the relative error in the determination of the illuminance is less than 5\%), as the point light source with anisotropic (cosine) luminous intensity and placed at their center, if the distance to the observation point is approximately four times larger than their characteristic sizes. It will be interesting in the future to generalize the obtained equations for the case of arbitrary orientation of the illuminated area.
1. M. Bukshtab, Photometry, Radiometry, and Measurements of Optical Losses (Springer, Singapore, 2019), p. 13, https: //doi.org/10.1007/978-981-10-7745-6

2. Zumbotel, The Lightning Handbook, Zumbotel, 2018.

3. J. B. Murdoch, Inverse Square Law Approximation of Illuminance, J. Illum. Eng. Soc. 10 (1981) 96, https://doi. org/10.1080/00994480.1980.10748595.

4. W. R. McCluney and R. McCluney, Introduction to Radiometry and Photometry (Boston, Artech House, 1994), pp. 11-15.
5. M. S. Rea, The IESNA lighting handbook (New York, IESNA, 2000), Ch. 9.

6. M. Bass et al., Handbook of Optics, 3rd ed. (McGraw-Hill, New York, 2009), Vol. 2.

7. M. J. Walker, The Treatment of Extended Light Sources in Elementary Textbooks, Am. J. Phys. 15 (1947) 65, https: //doi.org/10.1119/1.1990896 